\title{
Outcomes of combined trabecular micro-bypass and phacoemulsification in a predominantly Hispanic patient population
}

This article was published in the following Dove Press journal:

Clinical Ophthalmology

II October 2016

Number of times this article has been viewed

\author{
Mark J Gallardo',2 \\ Richard A Supnet' \\ Jane Ellen Giamporcaro ${ }^{3}$ \\ Dana M Hornbeak ${ }^{3}$ \\ 'El Paso Eye Surgeons, PA, El Paso, \\ ${ }^{2}$ Department of Ophthalmology, \\ University of Texas Health Science \\ Center at San Antonio, San Antonio, \\ TX, ${ }^{3}$ Division of Clinical Research and \\ Medical Affairs, Glaukos Corporation, \\ Laguna Hills, CA, USA
}

Purpose: The purpose of this study was to evaluate intraocular pressure (IOP) and topical ocular hypotensive medication burden at 12 months postoperatively in a predominantly Hispanic patient population with primary open-angle glaucoma each implanted with one trabecular micro-bypass stent during cataract surgery.

Methods: This was a retrospective, consecutive case series. The main objective was to assess reduction of IOP and/or medication burden in all eyes at the 12-month postoperative exam. A secondary objective was to assess outcomes in 3 subgroups, distinguished preoperatively by IOP control and by medication burden (suboptimal or maximum therapy) and with different treatment goals. Group 1 had medication-controlled IOP and goal to reduce medications while maintaining IOP control ( $\mathrm{n}=65$ ); Group 2 had uncontrolled IOP on $\leq 2$ medications and goal to reduce IOP and maintain/reduce medication burden $(n=31)$; and Group 3 had uncontrolled IOP on $\geq 3$ medications and goal to reduce IOP and avoid filtering surgery $(n=38)$. Evaluations included IOP, medication use, cup-to-disc ratio, visual fields, complications, and interventions. One hundred subjects (134 eyes) have been followed for 12 months.

Results: Most patients (80\%) were Hispanic and had moderate or severe glaucoma (87\%). At 12 months, mean IOP reduced to $12.9 \mathrm{mmHg}$ vs $16.5 \mathrm{mmHg}$ preoperatively; $92 \%$ had an IOP $\leq 15 \mathrm{mmHg}$ at 12 months $(99 \% \mathrm{had} \leq 18 \mathrm{mmHg})$. Mean medication burden had decreased to 0.9 vs 2.3 preoperatively. At the 12 -month time point, $94 \%$ of all eyes achieved their predefined treatment goal of reduced IOP and/or medications. Reductions in medication burden for Group 1, and in IOP for Groups 2 and 3, were highly statistically significant $(P<0.001)$. Two eyes in Group 3 had filtering surgery; the remaining $95 \%$ avoided such treatment. No other complications were reported.

Conclusion: This mainly Hispanic population with predominantly moderate or severe glaucoma had substantial reduction of IOP and medication and favorable safety for 12 months following stent implantation during cataract surgery, with treatment success achieved in all 3 subgroups. These data show this stent technology to be effective in Hispanic eyes with more advanced disease.

Keywords: glaucoma, trabecular micro-bypass, Hispanic, micro-invasive glaucoma surgery, IOP

\section{Introduction}

Glaucoma, a progressive degenerative disease causing optic nerve damage, is one of the leading causes of blindness worldwide. Currently affecting approximately 60 million people worldwide, it is expected to impact 78 million people by the year $2020 .{ }^{1}$ Until recently, glaucoma treatment has been limited to medications, laser treatment, and incisional surgery. However, the compliance issues and complications associated with 
these interventions can hamper their therapeutic effect, and in some cases may eliminate future treatment options. ${ }^{2}$ The development of ab interno trabecular micro-bypass stents for use during micro-invasive glaucoma surgery has significantly changed this treatment landscape. Trabecular micro-bypass stent implantation during cataract surgery has been shown to safely reduce both intraocular pressure (IOP) and medication usage through up to 5 years postoperatively in glaucoma patients. ${ }^{3-10}$ Furthermore, this treatment modality has been associated with fewer complications than those experienced after traditional incisional glaucoma surgery ${ }^{2}$ or more recent suprachoroidal stent procedures. ${ }^{11,12}$

To date, reports of trabecular micro-bypass stent implantation in conjunction with cataract surgery have involved patients with mild-to-moderate open-angle glaucoma (OAG) in predominantly non-Hispanic populations. Currently, Hispanics comprise $17.4 \%$ of the US population, and projections expect this percentage to rise to $28.6 \%$ by $2060 .{ }^{13}$ This population has been found to have a higher incidence and prevalence of OAG compared to Whites, with up to $21 \%$ of Hispanics developing OAG by the age of 80 years. ${ }^{14}$

The main goal of the current study was to assess postoperative IOP and medication reduction 12 months after combined trabecular micro-bypass stent implantation and cataract surgery in a primarily Hispanic patient population suffering from mostly moderate-to-severe OAG with varying degrees of IOP control while on either suboptimal or maximum tolerated medical therapy. A second objective was to assess outcomes in each of 3 subgroups as compared to their preoperative treatment goal. This report summarizes IOP, medication use, and safety data through 12 months postoperatively.

\section{Materials and methods Study design}

This was a retrospective, non-randomized, consecutive case series of 168 eyes of 128 patients with primary open-angle glaucoma (POAG) who underwent combined phacoemulsification cataract extraction and implantation of a single trabecular micro-bypass stent (iStent ${ }^{\circledR}$ Trabecular Micro-Bypass; Glaukos Corporation, Laguna Hills, CA, USA) (Figure 1). Surgeries were completed by a single surgeon (MG) over a 2-year period at an outpatient surgery center in El Paso, TX, USA. All patients were required to have POAG and cataract to be eligible for cataract surgery. Additional inclusion criteria included normal angle anatomy and absence of intraocular inflammation. Patients were excluded if they had corneal opacities preventing gonioscopic view, narrow angles, or angle

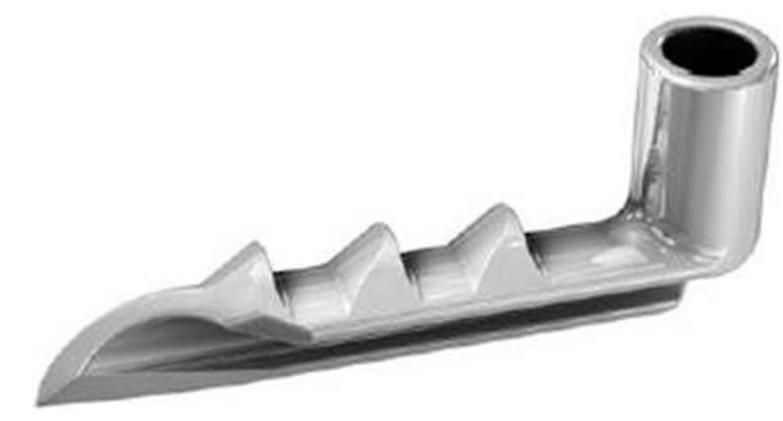

Figure I iStent ${ }^{\circledR}$ Trabecular Micro-Bypass.

closure. Patients were followed through 12 months postoperatively, and follow-up is ongoing. A total of 134 eyes of 100 patients had follow-up data through 1 year. Before surgery, eyes were placed into 3 groups by the surgeon according to his standard clinical practice for treating POAG. These groups were divided as follows, based on their treatment goal as well as level of IOP control (ie, IOP typically considered controlled if $\leq 18 \mathrm{mmHg}$ ) and/or medication burden (suboptimal therapy was 0-2 medications; maximum therapy was 3-4 medications). Group 1 included 65 eyes with IOP controlled on at least 1 medication, with the goal to reduce medications while maintaining IOP control. Group 2 included 31 eyes with IOP not controlled on $\leq 2$ medications, with the goal to reduce IOP and maintain or reduce medication burden. Of the 31 eyes, 9 eyes were on 0 medication due to medication intolerance, nonresponse, or newly diagnosed glaucoma. Group 3 comprised 40 eyes with uncontrolled IOP and/or on $\geq 3$ medications, with the goal to reduce IOP in order to avoid a filtering surgery. The choice of $18 \mathrm{mmHg}$ as the threshold for "uncontrolled" IOP was based on the AGIS study which showed minimal progression of visual field (VF) loss in eyes with IOP controlled to $<18 \mathrm{mmHg}$ over a 6 -year period. ${ }^{15}$ However, for patients with more severe glaucoma, medications were added to achieve lower IOP, as per the clinician's standard practice. Eyes considered to be "at risk" for a filtering surgery included those which would have undergone such surgery by MG in the past (prior to the development of iStent); this included all patients in Group 3, and certain patients in Group 2 who had progressive optic neuropathy and associated VF loss.

Preoperative and postoperative evaluations were completed by the staff surgeon and site staff, and included IOP, topical ocular hypotensive medication use, cup-to-disc (C:D) ratio, VFs via automated perimetry, and complications and interventions. Preoperative glaucoma severity was determined by VF mean deviation using the Hodapp-Parish-Anderson staging as mild ( 0 to $-6 \mathrm{~dB})$, moderate $(-6$ to $-12 \mathrm{~dB})$, and severe (worse than $-12 \mathrm{~dB}$ ). ${ }^{16}$ Data collection methods were 
followed in accordance with the Declaration of Helsinki, and all patients signed an informed consent to allow for the retrospective evaluation of their clinical data. Ethics approval was obtained from the Surgical Center of El Paso Medical Executive Committee (El Paso, TX, USA).

\section{Stent description, surgical technique, and perioperative medication}

The iStent is a titanium, heparin-coated, L-shaped stent approximately $1.0 \mathrm{~mm}$ in length and $0.33 \mathrm{~mm}$ in height, with a snorkel bore diameter of $120 \mu \mathrm{m}$. The iStent comes preloaded in a single-use, disposable, stainless steel inserter designed to facilitate ab interno stent insertion into Schlemm's canal. Once implanted, the stent body rests in the Schlemm's canal, and the snorkel resides in the anterior chamber.

Prior work by Samuelson et al has described the implantation technique in detail. ${ }^{3}$ In brief, the surgeon advances the iStent inserter through a temporal clear corneal incision after standard phacoemulsification and intraocular lens (IOL) implantation. Under gonioprism visualization, the stent is gently guided through the trabecular meshwork and into Schlemm's canal in the nasal portion of the aqueous drainage system. The stent is released by pushing the button on the inserter, and then the inserter is withdrawn.

At the end of the procedure, patients received a 1-week course of a topical antibiotic and a 4-week course of a topical nonsteroidal anti-inflammatory drug. If a persistent anterior chamber cellular reaction was identified at the Week 1 visit, a mild topical corticosteroid was prescribed 4 times a day for 1 week and tapered over a 6-day period.

Patients returned for postoperative follow-up examinations at Day 1, Week 1, and Months 1, 3, 6, and 12. Preoperative and postoperative evaluations included IOP, topical ocular hypotensive medication use, $\mathrm{C}: \mathrm{D}$ ratio, VFs, and complications and interventions.

\section{Data analyses}

Data analyses for the set of 134 eyes included assessment of mean (and standard deviation) IOP and number of medications used preoperatively and at Month 12 , and assessments of proportions of eyes with IOP $\leq 15 \mathrm{mmHg}$, IOP $\leq 18 \mathrm{mmHg}$, medications $\geq 3$, and medications $=0$. Best-corrected visual acuity (BCVA), IOP, C:D ratio, VFs via automated perimetry, and rates of complications and interventions were assessed through 12 months postoperatively. Within each treatment group, paired-sample $t$-tests were used to compare Month 12 change in IOP from preoperative IOP, and Wilcoxon signed-rank tests were used to compare Month 12 change in the number of medications from the preoperative number of medications. The significance level $(\alpha)$ was set at 0.05 . No adjustments were made for multiple comparisons.

\section{Results}

\section{Subject disposition and demographics}

A total of 167 eyes with visually significant cataracts and POAG were implanted with a single trabecular micro-bypass stent at the time of phacoemulsification cataract surgery. Of these 167 eyes, 134 had 1-year data, with the principle reason for absent data being loss to follow-up; there were also 2 patients within Group 3 who required secondary surgical intervention and thus were not included in the IOP and medication analyses (Table 1).

Table 2 summarizes the baseline demographic characteristics of this predominantly Hispanic study population. Preoperative glaucoma stage was severe (45\%), moderate (42\%), or mild (13\%) according to the Hodapp-ParishAnderson staging criteria (Table 3). ${ }^{16}$ Fourteen eyes had undergone 17 prior glaucoma surgeries, including selective laser trabeculoplasty $(n=8)$, laser peripheral iridotomy $(n=4)$, trabeculectomy $(n=2)$, Express ${ }^{\circledR}$ (Alcon Laboratories, Fort Worth, TX) shunt $(\mathrm{n}=2)$, and Ahmed implant $(\mathrm{n}=1)$.

\section{IOP and medication use}

When analyzing all 134 eyes with data through Month 12 collectively, 94\% were found to be able to achieve reduced IOP and/or medication burden after surgery. Mean IOP reduced to $12.9 \pm 2.1 \mathrm{mmHg}$ from $16.5 \pm 3.7 \mathrm{mmHg}$ preoperatively; $92 \%$ of eyes had an IOP $\leq 15 \mathrm{mmHg}$, and $99 \%$ had an IOP $\leq 18 \mathrm{mmHg}$ (vs $40 \% \leq 15 \mathrm{mmHg}$ and $75 \% \leq 18 \mathrm{mmHg}$ preoperatively; Figure 2). The medication burden dropped to $0.9 \pm 1.2$ from $2.3 \pm 1.1$ medications preoperatively. At 12 months, $17 \%$ of eyes were on $\geq 3$ medications vs $56 \%$ of eyes preoperatively; $54 \%$ were medication-free compared to $7 \%$ preoperatively. Further, the subset of eyes that were not on medication preoperatively continued to be medication-free

Table I Patient accountability at 12 months

\begin{tabular}{ll}
\hline & $\mathbf{n}(\%)$ \\
\hline Eyes with preoperative data & $\mathrm{I} 67(100)$ \\
Eyes with I-year data & $\mathrm{I} 34(80.2)$ (I00 subjects) \\
Eyes not available for analysis & $33(19.8)$ \\
Reasons not available for analysis & \\
$\quad$ Lost to follow-up & $27(16.2)$ \\
Deceased (nonsurgically related) & $2(1.2)$ \\
Secondary surgical intervention & $2(1.2)$ Group 3 patients \\
Moved to different city & $\mathrm{I}(0.6)$ \\
Hospice care & $\mathrm{I}(0.6)$ \\
\hline
\end{tabular}


Table 2 Patient demographics (100 subjects)

$80 \%$ Hispanic
$14 \%$ White
$6 \%$ Black
$61 \%$ female
$37 \%$ male
Mean age $74.6 \pm 8.9$ years old (median 75 , range $43-100$ )

at Month 12. Of the 46 eyes considered to be at risk for a filtering procedure, $44(96 \%)$ were able to avoid such treatment through 12 months postoperatively.

Group 1 included 65 eyes with controlled IOP on at least 1 medication and goal to reduce medications. At Month 12 , mean medication burden was $0.6 \pm 1.1$ vs $2.4 \pm-0.9$ preoperatively, a statistically significant reduction $(P<0.001$; Figure 3). Postoperatively, 69\% of eyes were medicationfree, and $11 \%$ were on $\geq 3$ medications (vs $57 \%$ preoperatively). In addition, IOP was not only maintained but also actually decreased slightly, to $12.7 \pm 1.9 \mathrm{mmHg}$ at 12 months from $13 . \pm 1.8 \mathrm{mmHg}$ preoperatively. The mean IOP change from preoperative value at 12 months was $-0.88 \mathrm{mmHg}$ with a $95 \%$ confidence interval of $(-1.43,-0.32) \mathrm{mmHg}$.

Group 2 included 31 eyes with IOP not controlled on $\leq 2$ medications and the goal to reduce IOP. When analyzing this group individually, $100 \%$ of eyes had reduced IOP at 12 months. Mean IOP decreased to $12.6 \pm 2.1 \mathrm{mmHg}$ from $19.1 \pm 2.4 \mathrm{mmHg}$ preoperatively. This reduction in IOP was statistically significant $(P<0.001$; Figure 4). Further, all eyes had an IOP $\leq 18 \mathrm{mmHg}$ at 12 months vs $45 \%$ of eyes at this level preoperatively. In addition to this IOP reduction, medications decreased to $0.4 \pm 0.7$ from $1.1 \pm 0.8$ preoperatively. While only $29 \%$ of eyes were medicationfree preoperatively, $68 \%$ were medication-free at 12 months, with no eyes increasing the number of medications from preoperative number.

Table 3 Preoperative ocular parameters (134 eyes)

\begin{tabular}{ll}
\hline Eye & $42 \%$ OD, 58\% OS \\
Glaucoma severity & $45 \%$ severe \\
& $42 \%$ moderate \\
& $13 \%$ mild \\
Cup:disc ratio, mean \pm SD & $0.7 \pm 0.2$ \\
Visual field (mean deviation), mean \pm SD & $-9.1 \pm 8.3 \mathrm{~dB}$ \\
Prior surgeries ( $\mathrm{n})$ & $\mathrm{I}(\mathrm{I}$ eyes $)$ \\
SLT & $\mathrm{n}=8$ \\
LPI & $\mathrm{n}=4$ \\
Trabeculectomy & $\mathrm{n}=2$ \\
Express ${ }^{\circledR}$ shunt & $\mathrm{n}=2$ \\
Ahmed implant & $\mathrm{n}=1$ \\
\hline
\end{tabular}

Abbreviations: OD, oculus dexter; OS, oculus sinister; SD, standard deviation; SLT, selective laser trabeculoplasty; LPI, laser peripheral iridotomy.

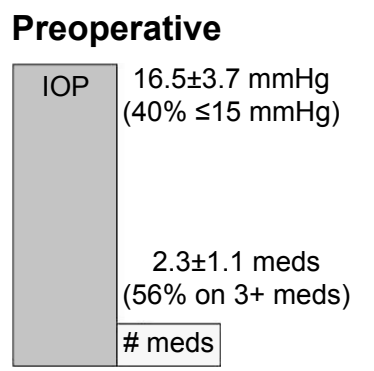

\section{M12 postoperative}

$94 \%$ had reduced IOP and/or medication at Month 12

Figure 2 Preoperative vs Month 12 postoperative IOP and medication use - all eyes $(n=134)$.

Abbreviations: IOP, intraocular pressure; MI2, Month I2; meds, medications.

Group 3 included eyes with uncontrolled IOP and/or on $\geq 3$ medications, with the goal to reduce IOP to avoid a filtering surgery. Of the original 40 eyes in this group, $95 \%$ (38 of the 40 eyes) achieved their predefined treatment goal of reduced IOP and avoidance of filtering surgery. In these eyes, mean IOP decreased to $13.6 \pm 2.3 \mathrm{mmHg}$ from $19.3 \pm 3.1 \mathrm{mmHg}$ (statistically significant reduction; $P<0.001$ ), with $97 \%$ of eyes with IOP $\leq 18 \mathrm{mmHg}$ vs $55 \%$ preoperatively (Figure 5). Medication burden decreased to $1.8 \pm 1.2$ from $3.2 \pm 0.4$ preoperatively. While $100 \%$ of eyes were on $\geq 3$ medications preoperatively (with $16 \%$ on $4+$ medications), $42 \%$ were on this level at 12 months. Two eyes $(5 \%)$ required secondary surgical intervention (filtration surgery using an Express device), but the remaining 38 eyes (95\%) were able to avoid filtering surgery through 12 months postoperatively.

\section{Safety assessment}

No intraoperative ocular adverse events occurred, including possible events such as hyphema, iris damage, corneal injury, or failure to implant the stent or IOL. For all eyes collectively, as well as for each of the 3 groups, C:D ratio and VF (mean deviation) values remained similar at 12 months postoperatively vs preoperative levels. Month 12 BCVA

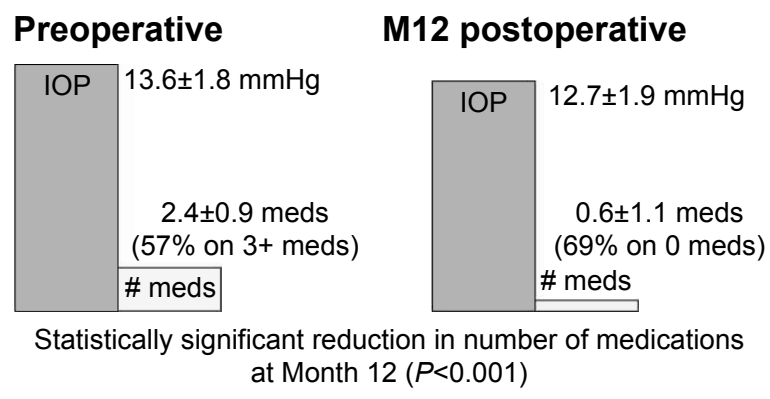

Figure 3 Preoperative vs Month 12 postoperative IOP and medication use Group I (n=65).

Abbreviations: IOP, intraocular pressure; MI2, Month 12; meds, medications. 


\section{Preoperative

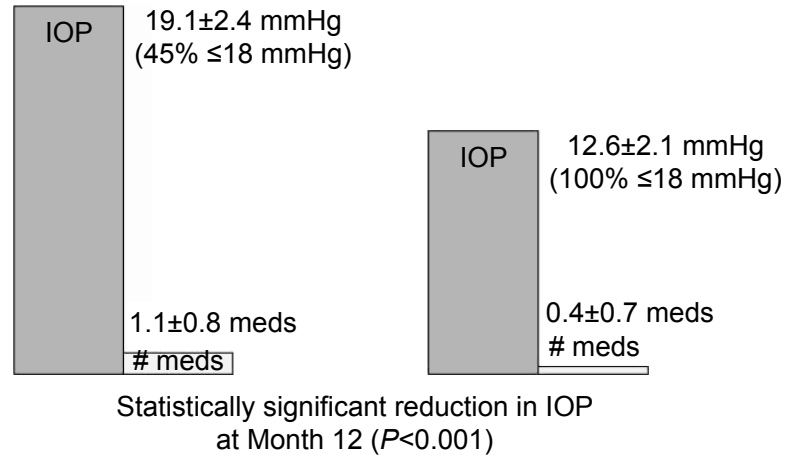

Figure 4 Preoperative vs Month 12 postoperative IOP and medication use - Group 2 $(n=31)$.

Abbreviations: IOP, intraocular pressure; MI2, Month 12; meds, medications.

improved, such that $83 \%$ of eyes achieved a BCVA of $20 / 40$ or better after surgery (vs $20 \%$ preoperatively) and $8 \%$ achieved a BCVA of 20/50 to 20/80. The 12 eyes with Month 12 BCVA of 20/100 or worse had severe glaucoma and 20/100 or worse BCVA preoperatively. Two patients in Group 3 required secondary surgical intervention (filtration surgery using an Express device). No other adverse events were reported.

\section{Discussion}

In this group of predominantly Hispanic patients, $86.5 \%$ of whom had moderate-to-severe glaucoma and over half of whom had a substantial preoperative medication burden, results 1 year following implantation of 1 iStent during cataract surgery showed treatment success in $94 \%$ of all eyes vs original treatment goal of reduced IOP and/or medications. Clinical findings included reduction in mean IOP to $12.9 \pm 2.1 \mathrm{mmHg}$ vs $16.5 \pm 3.7 \mathrm{mmHg}$ preoperatively, with $92 \%$ of eyes with an IOP $\leq 15 \mathrm{mmHg}$ and $99 \%$ with an

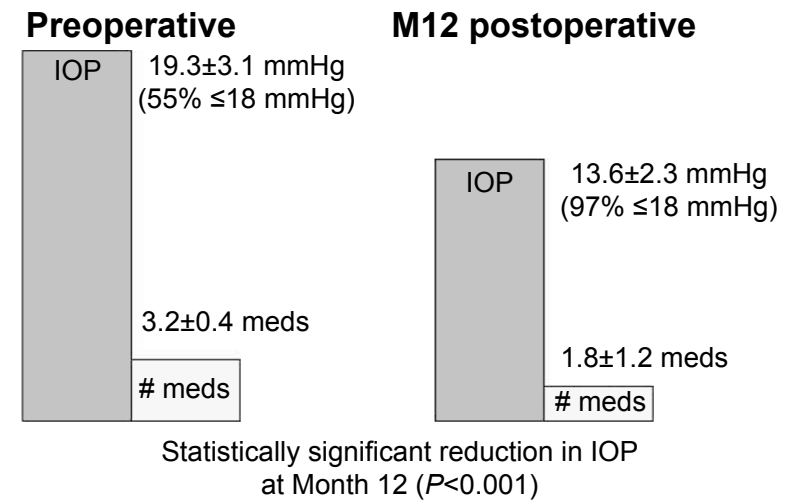

Figure 5 Preoperative vs Month 12 postoperative IOP and medication use-Group 3 $(n=38)$.

Abbreviations: IOP, intraocular pressure; MI2, Month 12; meds, medications.
IOP $\leq 18 \mathrm{mmHg}$, and reduction in mean number of medications to 0.9 vs 2.3 preoperatively.

Considering the higher incidence and prevalence of OAG development in Hispanic patients vs Caucasian patients,,${ }^{14}$ the present study showing clinical outcomes of iStent-cataract surgery in a predominantly Hispanic population fills a key gap in the literature. Prior studies have demonstrated the effectiveness and safety of trabecular micro-bypass stent implantation in conjunction with cataract surgery for mild-to-moderate glaucoma in predominantly Caucasian cohorts. ${ }^{3-10}$ There is a relative paucity of glaucoma studies in Hispanic patients, who currently comprise $17.4 \%$ of the US population and are estimated to increase by $64 \%$ over the next half-century. ${ }^{13}$

In addition, the present study focuses on more advanced stages of glaucoma than most prior studies of iStent outcomes. In these more advanced glaucoma patients, treatment success (as indicated by IOP and/or medication reduction) was achieved across all 3 predefined surgical goals In particular, $96 \%$ of eyes at risk for a filtering procedure were able to avoid such treatment through 1 year postoperatively. Taking into account the postoperative infection rates and other complications, and long-term follow-up (eg, bleb needling, etc.) associated with filtering surgery, ${ }^{2}$ as well as the commensurate considerable health care costs, ${ }^{17}$ avoidance of such surgery may offer improved patient quality of life as well as significant cost savings.

Furthermore, the study was completed in a real-life clinical setting, where the goals of iStent surgery may differ based on the patient and disease state. For example, for patients in Group 1 (ie, patients with controlled IOP who would benefit from fewer medications), treatment success may be defined by reduced medication burden rather than reduced IOP. This medication reduction is important, given the notable increase in adherence rates when patients go from multiple eyedrop bottles to only 1 bottle, ${ }^{18-20}$ as well as the considerable costs of generic and brand-name topical medications. ${ }^{21}$ Since the majority of glaucoma patients have at least 1 additional chronic condition requiring medication, ${ }^{22}$ a reduction in number of medications would be beneficial for both adherence and cost. Alternatively, for patients in Group 2 (ie, patients with uncontrolled IOP despite using $\leq 2$ medications, or patients with uncontrolled IOP who are intolerant to topical medications), treatment success may be more accurately defined by reduced IOP rather than reduced medications. Given this real-life clinical population, this study's high success rate - both in the overall set of eyes and in the different subgroups - is an important finding. 
The safety data were very favorable, with no patients experiencing complications or adverse events associated with conventional glaucoma filtering procedures such as endophthalmitis, bleb formation, hypotony, and fibrosis. In addition, no patients experienced the complications reported to occur at a clinically significant rate with more invasive suprachoroidal stent procedures, such as peripheral anterior synechiae, inflammation, hypotony, and implant obstruction. ${ }^{11,12}$ In our study, 2 patients at risk of filtering surgery required additional surgical intervention (filtration surgery using an Express device). Overall VF measures and $\mathrm{C}: \mathrm{D}$ ratio did not appear different from preoperative values, and improvement of BCVA after cataract surgery was maintained through 12 months postoperatively. There are several limitations to this retrospective, single-site study. One inherent artifact of retrospective analysis is the absence of a control group. Previous work has compared outcomes of stent placement with and without cataract surgery and has shown a treatment effect for stent-cataract surgery vs cataract surgery alone. ${ }^{3,4}$ In contrast, the goal of our study was to assess outcomes in a novel patient population (primarily Hispanic) and in a more advanced stage of glaucoma. Although we did not include a concurrent control group of cataract surgery alone, we considered preoperative IOP to be a valid control for comparison, as IOP is an objective numeric measure.

Another artifact of the retrospective design is that preoperative and postoperative medication washouts were not completed, as these are not customarily done in standard clinical practice and could place at risk patients with moderate or severe disease such as those in our study. Twelvemonth postoperative data were not available for each patient; and diurnal IOP measurements were not measured. Patients did not fulfill specific inclusion criteria, but rather comprised a consecutive series from the author's clinical practice. Finally, this report summarizes data at 12 months. A future report from this study can encompass events at various time points vs only 1 postoperative time point, as well as longer postoperative follow-up. In addition, future analyses can further stratify outcomes by ethnicity, surgical goals, and history of filtering surgery.

In summary, not only does this study show the value of using stent technology to safely reduce IOP and medication burden, but it also does so across a demographically diverse patient population with more advanced glaucoma and substantial need for better glaucoma control.

\section{Disclosure}

This study was presented in part at the 2016 annual meeting of the American Glaucoma Society and the 2016 annual meeting of the American Society of Cataract and Refractive Surgeons. Dr Gallardo is a consultant and speaker for Glaukos Corporation, Laguna Hills, CA, USA. Jane Ellen Giamporcaro and Dana Hornbeak are employees of Glaukos. Richard Supnet has no conflicts of interest to report in this work.

\section{References}

1. Quigley HA. Glaucoma. Lancet. 2011;377(9774):1367-1377.

2. Rulli E, Biagioli E, Riva I, et al. Efficacy and safety of trabeculectomy vs nonpenetrating surgical procedures: a systematic review and metaanalysis. JAMA Ophthalmol. 2013;131(12):1573-1582.

3. Samuelson TW, Katz LJ, Wells JM, Duh YJ, Giamporcaro JE; US iStent Study Group. Randomized evaluation of the trabecular micro-bypass stent with phacoemulsification in patients with glaucoma and cataract. Ophthalmology. 2011;118(3):459-467.

4. Fea AM. Phacoemulsification versus phacoemulsification with microbypass stent implantation in primary open-angle glaucoma. $J$ Cataract Refract Surg. 2010;36(3):407-412.

5. Belovay GW, Naqi A, Chan BJ, Rateb M, Ahmed II. Using multiple trabecular micro-bypass stents in cataract patients to treat open-angle glaucoma. J Cataract Refract Surg. 2012;38(11):1911-1917.

6. Craven ER, Katz LJ, Wells JM, Giamporcaro JE; iStent Study Group. Cataract surgery with trabecular micro-bypass stent implantation in patients with mild-to-moderate open-angle glaucoma and cataract: two-year follow-up. J Cataract Refract Surg. 2012;38(8):1339-1345.

7. Arriola-Villalobos P, Martinez-de-la-Casa J, Diaz-Valle D, et al. Combined iStent trabecular micro-bypass stent implantation and phacoemulsification for coexistent open-angle glaucoma and cataract: a long-term study. Br J Ophthalmol. 2012;96(5):645-649.

8. Neuhann TH. Trabecular micro-bypass stent implantation during small-incision cataract surgery for open-angle glaucoma ocular hypertension: long-term results. J Cataract Refract Surg. 2015;41(1): 2664-2671.

9. Fea AM, Consolandi G, Zola M, et al. Micro-Bypass implantation for primary open-angle glaucoma combined with phacoemulsification: 4-year follow-up. J Ophthalmol. 2015;2015:795357.

10. Spiegel D, Wetzel W, Neuhann T, et al. Coexistent primary openangle glaucoma and cataract: interim analysis of a trabecular microbypass stent and concurrent cataract surgery. Eur J Ophthalmol. 2009; 19(3):393-399.

11. Höh H, Grisanti S, Grisanti S, Rau M, Ianchulev S. Two-year clinical experience with the CyPass micro-stent: safety and surgical outcomes of a novel supraciliary micro-stent. Klin Monbl Augenheilkd. 2014;231(4):377-381.

12. Sarkisian S. Combined cataract surgery and supraciliary microstent implantation for open-angle glaucoma: multicenter 3-year results. Presented at: 2016 American Society of Cataract and Refractive Surgeons; May 7, 2016; New Orleans, LA.

13. Colby SL, Ortman JM. Projections of the size and composition of the U.S. population: 2014 to 2060 . Current population reports. P25-1143. Washington, DC: U.S. Census Bureau; 2014.

14. Varma R, Ying-Lai M, Francia BA, et al; Los Angeles Latino Eye Study Group. Prevalence of open-angle glaucoma and ocular hypertension in Latinos: the Los Angeles Latino Eye Study. Ophthalmology. 2004;111(8):1439-1448.

15. AGIS Investigators. The Advanced Glaucoma Intervention Study (AGIS): 7. The relationship between control of intraocular pressure and visual field deterioration. Am J Ophthalmol. 2000;130(4):429-440.

16. Hodapp E, Parrish RK II, Anderson DR. Clinical Decisions in Glaucoma. St Louis, MO: The CV Mosby Co; 1993.

17. Lee PP, Kelly SP, Mills RP, et al; Costs of Glaucoma Study Group. Glaucoma in the United States and Europe: predicting costs and surgical rates based upon stage of disease. J Glaucoma. 2007;16(5):471-478.

18. Schwartz GF, Quigley HA. Adherence and persistence with glaucoma therapy. Surv Ophthalmol. 2008;53 Suppl 1:S57-S68. 
19. Djafari F, Lesk MR, Harasymowycz PJ, Desjardins D, Lachaine J. Determinants of adherence to glaucoma medical therapy in a long-term patient population. J Glaucoma. 2009;18(3):238-242.

20. Robin AL, Covert D. Does adjunctive glaucoma therapy affect adherence to the initial primary therapy? Ophthalmology. 2005;112(5):863-868.
21. Red Book ${ }^{\mathrm{TM}}$. Ann Arbor, MI: Truven Health Analytics; 2013. Available from: http://micromedex.com/products/product-suites/clinicalknowledge/redbook. Accessed July 6, 2016.

22. Salim S, Shields MB. Glaucoma and systemic diseases. Surv Ophthalmol. 2010;55(1):64-77.

\section{Publish your work in this journal}

Clinical Ophthalmology is an international, peer-reviewed journal covering all subspecialties within ophthalmology. Key topics include: Optometry; Visual science; Pharmacology and drug therapy in eye diseases; Basic Sciences; Primary and Secondary eye care; Patien Safety and Quality of Care Improvements. This journal is indexed on

Submit your manuscript here: http://www.dovepress.com/clinical-ophthalmology-journal

\section{Dovepress}

PubMed Central and CAS, and is the official journal of The Society of Clinical Ophthalmology (SCO). The manuscript management system is completely online and includes a very quick and fair peer-review system, which is all easy to use. Visit http://www.dovepress.com/ testimonials.php to read real quotes from published authors. 